\title{
Factores predictores del compromiso docente en escuelas públicas peruanas: un modelo estructural
}

\author{
Consuelo Cerviño \\ Irene Fernández \\ José M. Tomás \\ Universitat de València, Valencia, España \\ Susana Córdova \\ Ministerio de Educación, Lima, Perú
}

Recibido: 12 de abril del 2016 / Aceptado: 03 de junio del 2016

El compromiso docente con los procesos de cambio tiene lugar en un contexto en el que intervienen sus propias actitudes y conceptos sobre la educación. Por tanto, el cambio educativo necesita del compromiso de los docentes, puesto que, para que este sea exitoso, es imprescindible que el docente replantee la profesión. Los factores personales y los profesionales intervienen en los procesos de cambio docente. Por consiguiente, el objetivo fue poner a prueba un modelo estadístico predictivo del compromiso docente con el cambio educativo a partir de factores de personalidad y autoevaluación. La muestra, aleatoria y representativa de la población de docentes peruanos, fue de 888 . El modelo resultó en un ajuste adecuado, con un gran poder predictivo sobre las dimensiones de procesos de cambio. En suma, el modelo confirma que variables personales, como son el autoconcepto y la personalidad, tienen fuerte poder predictivo sobre el compromiso docente.

compromiso docente /cambio educativo / personalidad / autoconcepto

\section{Predictive Factors in Teachers' Engagement in Change Processes in Peruvian Public Schools: A Structural Model}

Teachers' engagement in change processes is closely related to their attitudes and views on education. Therefore, any successful educational change demands that teachers reevaluate their profession, as personal and professional factors are clearly relevant. The goal of this study is to test a predictive statistical model of teacher's engagement in educational change considering personality factors and self-evaluation. The random and representative sample of Peruvian teachers comprised of 888 subjects. Results show good model fit and a strong predictive power on educational changes. The study confirms that personality and selfconcept are strong predictors on teacher's engagement with educational change.

teacher engagement / educational change / personality / self-concept

Correo electrónico: ifermar2@alumni.uv.es 


\section{INTRODUCCIÓN}

La labor de los docentes no se reduce a la vida en el aula, sino que va más allá del ámbito escolar y busca transformar las estructuras sociales en que se embebe la actividad académica (Martínez, 2008). Los docentes deben ser profesionales comprometidos con el cambio y, por tanto, todo docente debe contribuir con el cambio social y educativo. Aunque existan resistencias al cambio, es necesario que la gestión de este transforme el sistema educativo involucrando a todos los profesores (Tovar, 2009). Las políticas sociales docentes favorecen el cambio tanto del docente como de la educación (Tedesco y López, 2013), ya que aquellos profesores que se perciban en una situación de ascenso social serán los más predispuestos a asumir compromisos frente a nuevos desafíos. Por otro lado, aquellos que perciben que sus condiciones laborales se han deteriorado con el paso del tiempo, forman el grupo más resistente a las propuestas de reforma. El compromiso docente con los procesos de cambio tiene lugar en un contexto en el que intervienen, por tanto, sus propios conceptos sobre la educación. El cambio educativo necesita del compromiso de los docentes, puesto que para que este sea exitoso, es imprescindible que el docente replantee la profesión (Tedesco y López, 2013). Además, según Monereo (2010), los factores personales y los profesionales intervienen en los procesos de cambio docente. Así, estos factores contribuirán a que los docentes se comprometan en dichos procesos de cambio.
Uno de estos factores personales es la personalidad del profesor. Rapoport (1997) vincula los roles laborales con las características de personalidad y sostiene, por ejemplo, que personas altas en escrupulosidad preferirán instituciones en las que personas con otro perfil de personalidad se sentirían fracasadas. En cuanto a los factores que maximizan la formación y el desempeño óptimo en el ámbito profesional, Del Buey et al., (2008) identifican, a partir de la literatura, factores como estabilidad emocional (bajos niveles de neuroticismo), apertura a la experiencia, extraversión y responsabilidad. En cambio, otros autores han encontrado que el neuroticismo, la extraversión y la responsabilidad tenían un efecto significativo, mientras que la amabilidad y la apertura a la experiencia mostraban efectos no significativos (Judge, Heller y Mount, 2002). En el caso de la profesión docente, el efecto de los factores de la personalidad sobre el desarrollo de la profesión adquiere vital importancia, ya que las características del profesor actuarán sobre los alumnos y su aprendizaje (Peiró, 1987).

Los rasgos de personalidad forman las bases para la existencia de patrones conductuales relativamente consistentes a lo largo del tiempo. Estos patrones se mantienen unidos a través del autoconcepto. Este está formado por las creencias y opiniones del individuo sobre sí mismo, la evaluación del individuo sobre sí mismo y las relaciones interpersonales derivadas de estos dos componentes (Llor, Abad, García y Nieto, 1995). Según Gómez (2007), el desempeño depende del 
nivel de competencia percibido por uno mismo. Esto tiene implicaciones académicas en la labor docente, puesto que, si los profesores tienen mayor confianza en su capacidad personal y profesional para afrontar el trabajo, pueden alcanzar resultados más exitosos. La competencia percibida es un juicio acerca de la capacidad de uno mismo para llevar a cabo las acciones necesarias para cumplir un determinado objetivo.

Por otro lado, la valoración de uno mismo, a través de una buena motivación creará una conducta exitosa La aceptación y valoración de uno mismo contribuye al desarrollo profesional y al cambio de conducta, lo que desemboca en un mejor desempeño profesional. Un alto autoconcepto del docente, especialmente el autoconcepto académico, se encuentra muy ligado a la satisfacción laboral, la cual tiene implicaciones en el desempeño profesional. Más concretamente, se ha visto que una mayor satisfacción laboral por parte de los docentes está relacionada con un mayor rendimiento en la escuela (Chamundeswari, 2013). Este rendimiento puede verse como una consecuencia de un mayor nivel de compromiso (Kompaso y Sridevi, 2010).

Otro factor comprendido en el autoconcepto es la interacción con los otros, que es fuente de desarrollo para este, especialmente por la información que aportan estas personas de nosotros mismos (Cava y Musitu, 2000). En el caso de los docentes, estas personas incluyen los otros colegas docentes, los alumnos, los padres de estos y la comunidad educativa.
Los maestros se enfrentan a situaciones complejas en sus relaciones con otras personas, que suponen una oportunidad para el aprendizaje y crecimiento personal. Cabe enfatizar la relación profesor-alumno, ya que el docente tiene un efecto en el estudiante $y$, cuando este es positivo, puede potenciar un rendimiento más alto (Burns, 1990). En general, los profesores con un buen autoconcepto suelen ser percibidos como más eficaces, así como mejor capacitados para la formación de los estudiantes. Este tipo de docentes orientan al alumno a la maestría y mantienen una actitud abierta al cambio.

Como se ha visto, el profesor juega un papel fundamental en el proceso educativo y tiene como objetivo contribuir a la formación de los estudiantes en todas las dimensiones del desarrollo humano. Desde esta perspectiva, transformar la educación es esencial para la formación de la sociedad (Consejo Nacional de Educación, 2007). Por tanto, el compromiso del docente con dicha transformación educativa es de vital importancia, y es necesario seguir esta línea de investigación con tal de aportar más información sobre los mecanismos que contribuyen al compromiso del docente con el cambio. En el presente estudio, se pretende poner a prueba un modelo estadístico predictivo del compromiso docente con el cambio educativo a partir de variables personales, y en concreto de los cinco grandes factores de personalidad y la percepción del sí mismo (autoconcepto) docente, en una muestra representativa de docentes peruanos. 


\section{Método}

\section{Diseño y muestra}

El diseño de investigación es correlacional y consiste en una encuesta transversal a una muestra de docentes peruanos que intenta ser representativa de la población docente peruana. El universo del estudio estuvo constituido por 1478 instituciones educativas (IIEE) públicas peruanas de la Educación Básica Regular, que cuentan con 10942 docentes de los niveles educativos de Educación Inicial, Educación Primaria y Educación Secundaria, ubicadas en la región Lima Metropolitana (costa), región Huancavelica (sierra) y región Loreto (selva). De este universo, se obtuvo una muestra aleatoria de 87 conglomerados, que dio lugar a una muestra total de 888 docentes. Esta muestra suponía una tasa máxima de error de $5 \%$, con un nivel de confianza del $95 \%$ y $\mathrm{p}=\mathrm{q}=.5$. En cuanto a los descriptivos de escuelas y docentes, el $36 \%$ de las escuelas muestreadas se situó en la selva; el $26 \%$, en la costa, y el restante $38 \%$, en la sierra. En cuanto a los docentes, el 63,9\% fue mujer. Los docentes hasta 35 años alcanzan el $30 \%$; los que tienen entre 36 y 45 años, el $42 \%$, y los mayores de 46 años, el $28 \%$. Por lengua materna, el 79,6\% era castellanohablante; el 18,2\% hablaba quechua; un $1,7 \%$, aymara, y un $0,2 \%$, el kukama-kukamiria y el shawi. Los docentes nacidos en la costa eran el 25,2\% del total; un $42,5 \%$, de la sierra, y el restante $32,23 \%$, de la selva. Un $27,9 \%$ era soltero; un $53 \%$, casado, y el resto se encontraba ubicado en otros estados civiles.
Finalmente, en cuanto a su situación laboral, los docentes nombrados eran un $78,6 \%$, y los contratados, un $21,4 \%$.

\section{Instrumentos de medida}

La investigación emplea indicadores y cuestionarios ya validados con anterioridad. En concreto, y además de indicadores sociodemográficos de los docentes y descriptivos de las instituciones educativas, se han empleado las siguientes escalas:

a) Cuestionario Percepción del sí mismo docente (CPSMD), creado por Calvete y Villa (1997). El alfa para los factores fue .84 (Competencia), .76 (Autorrealización), .77 (Percepción interpersonal), .69 (Satisfacción), .68 (Asunción de riesgos), .64 (Autoaceptación) y .74 (Relación con los alumnos).

b) Cuestionario NEO-FFI, Inventario de Personalidad (CNEOFFI). Este Inventario NEO reducido de Cinco Factores, de Costa y McCrae (2008), es una versión del NEO PI-R que permite una medida general y rápida de la personalidad. El alfa para las distintas dimensiones fue el siguiente: Neuroticismo $=.90$, Extraversión $=.84$, Apertura $=.82$, Amabilidad $=.83$ y Responsabilidad $=.88$.

c) Compromiso del docente (CCD), elaborado por Sánchez y Cerviño (2006), con una solución final de 22 ítems. La fiabilidad obtenida en los factores del CCD para el factor de Compromiso docente con los procesos de cambio fue de .88; para el Compromiso del docente con la transformación de 
la práctica educativa, .74; y para el Compromiso con la relación con la comunidad educativa, .70 .

\section{Análisis estadístico}

Se han calculado estadísticos descriptivos y coeficientes de correlación entre las dimensiones (factores) de la Percepción del sí mismo docente (CPSMD), el Compromiso del docente (CCD) y el Cuestionario NEO-FFI, Inventario de Personalidad (CNEOFFI), todos ellos calculados en SPSS 21. Se estima el efecto y las relaciones entre múltiples variables a través de modelos de ecuaciones estructurales, en particular mediante modelos estructurales con variables observables. Se parte de un modelo predictivo inicial completamente a priori, que luego puede ser o no confirmado como una buena representación de las relaciones entre las variables. Para estimar los modelos, se ha empleado máxima verosimilitud con correcciones robustas, el procedimiento recomendado para este tipo de datos (Finney y Di Stefano, 2006). Los modelos de ecuaciones estructurales descritos en este estudio se estimaron con EQS 6.1 (Bentler, 2005). Para comprobar el ajuste del modelo, se han utilizado varios índices de ajuste, lo recomendado en la literatura especializada (Hu y Bentler, 1999; Tanaka, 1993). En concreto, se han calculado (a) el estadístico chi-cuadrado $\left(\chi^{2}\right)$; (b) el Comparative Fit Index (CFI), cuyo punto de corte para considerar ajuste adecuado es de más de .90 (e idealmente más de .95); (c) Root Mean Square Error of Approximation (RMSEA), que se espera sea menor que .05; y (e) el Standardized Root Mean Squared Residuals (SRMR), que se considera indicativo de buen ajuste si vale igual o menos de $.08(\mathrm{Hu}$ y Bentler, 1999).

\section{Resultados}

La Tabla 1 muestra los coeficientes de correlación de Pearson entre todas las dimensiones del modelo estructural; esto es, entre Competencia docente, Autorrealización docente, Relación con alumnos, Satisfacción docente, Percepción interpersonal, Aceptación de sí mismo, Evitación de riesgos e iniciativas, Introversión, Neuroticismo, Responsabilidad, Apertura y Amabilidad, y las medidas de Compromiso docente (compromiso referido a los Procesos de cambio, la Transformación de la práctica educativa y la Relación con la comunidad educativa).

Los modelos estructurales empleados han sido modelos de path analysis o modelos estructurales con variables observables, que permiten estimar el efecto y relacionar las variables de interés estableciendo un modelo teórico a priori en el que se consideran todas las relaciones que se esperan entre estas. En este caso, fue sencillo, ya que consistió en un modelo predictivo simultáneo de las tres dimensiones del Compromiso docente sobre la base de las características personales de percepción del sí mismo del docente y sus características de personalidad. El modelo inicial teórico puede verse en la Figura 1. Este asumió que los predictores no serían independientes, salvo los factores de cada una de las escalas, pues eran ortogonales. 


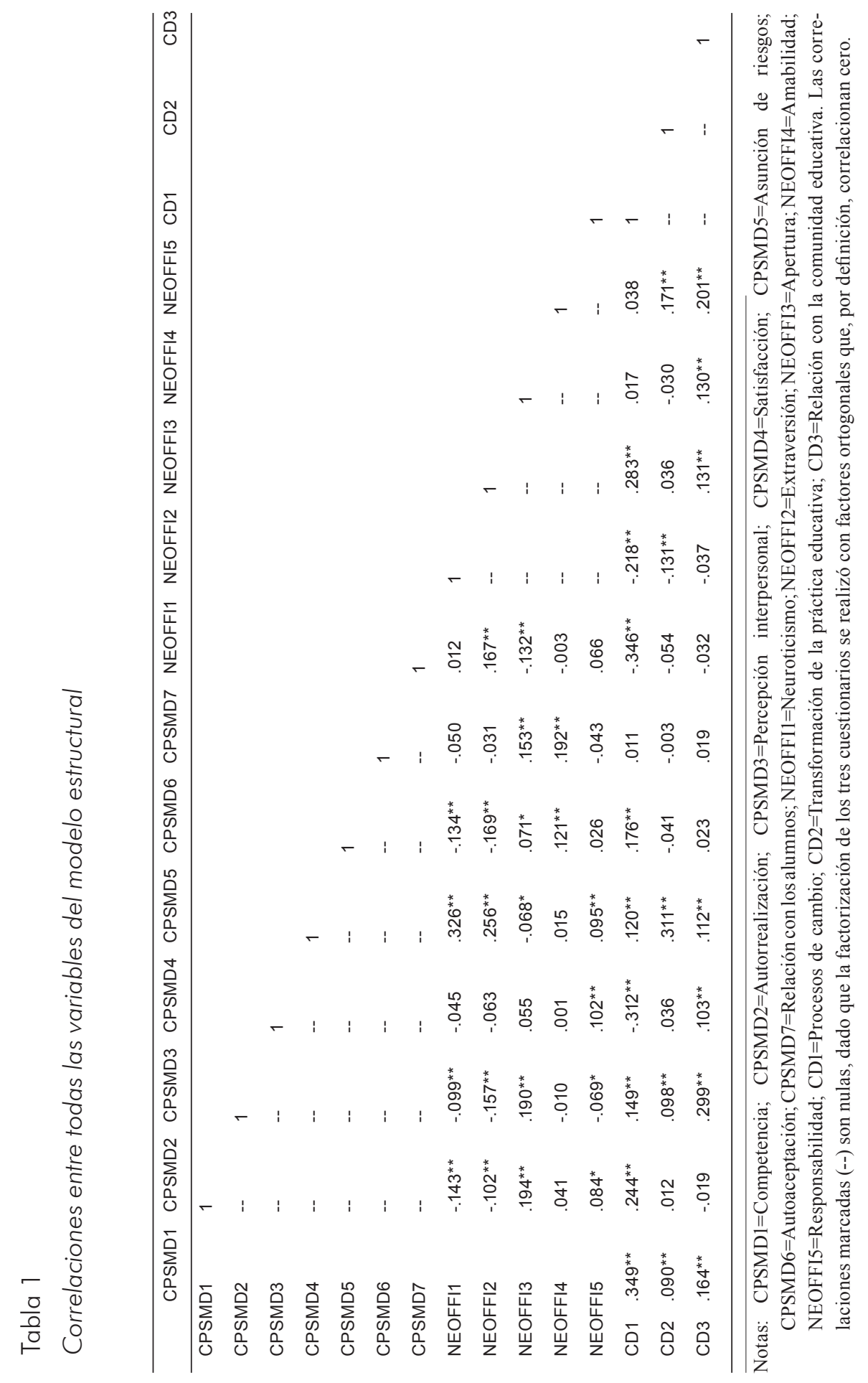




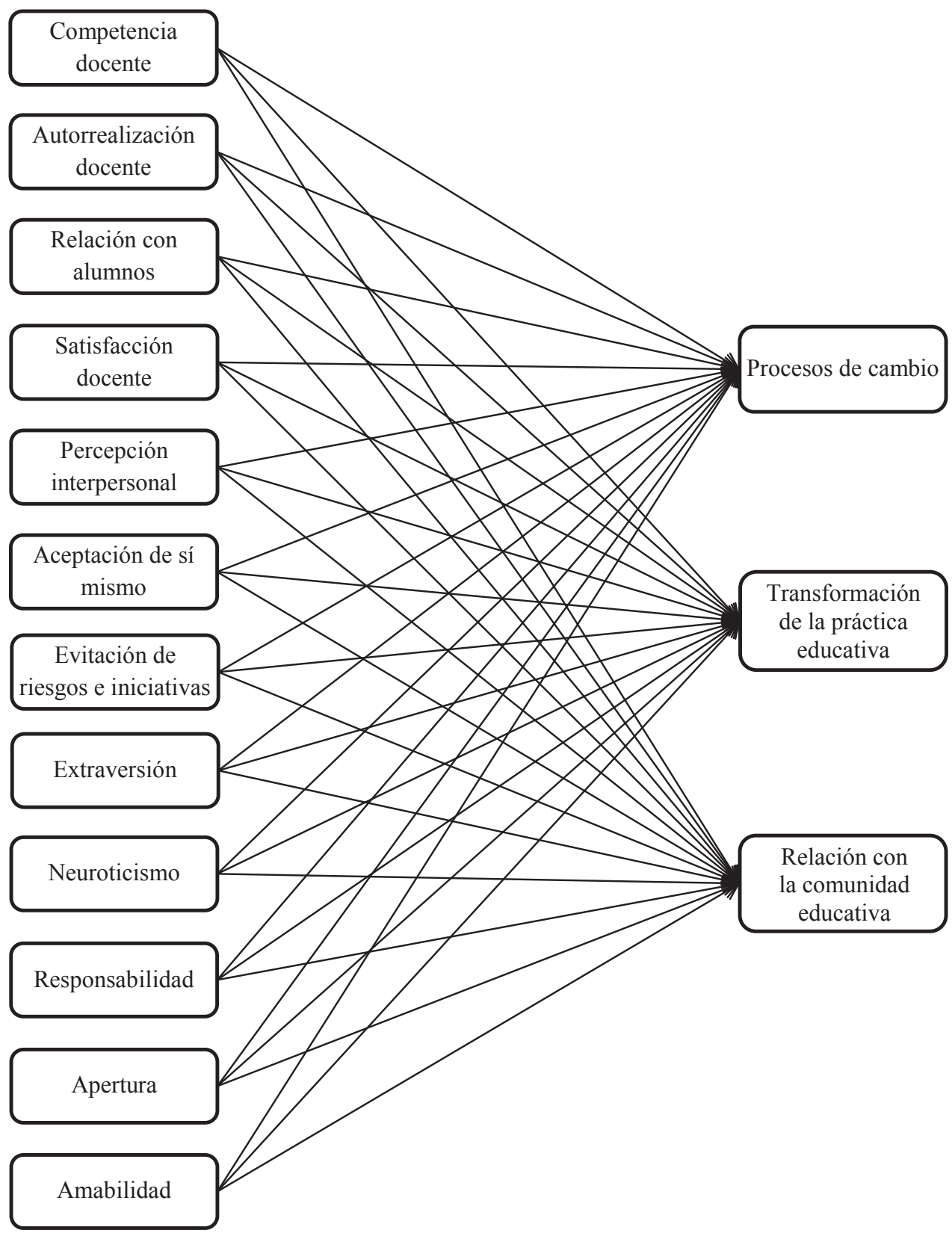

Figura 1 Modelo estructural inicial para predecir simultáneamente las tres medidas de Compromiso docente: Procesos de cambio, Transformación de la práctica educativa y Relación con la comunidad educativa. 


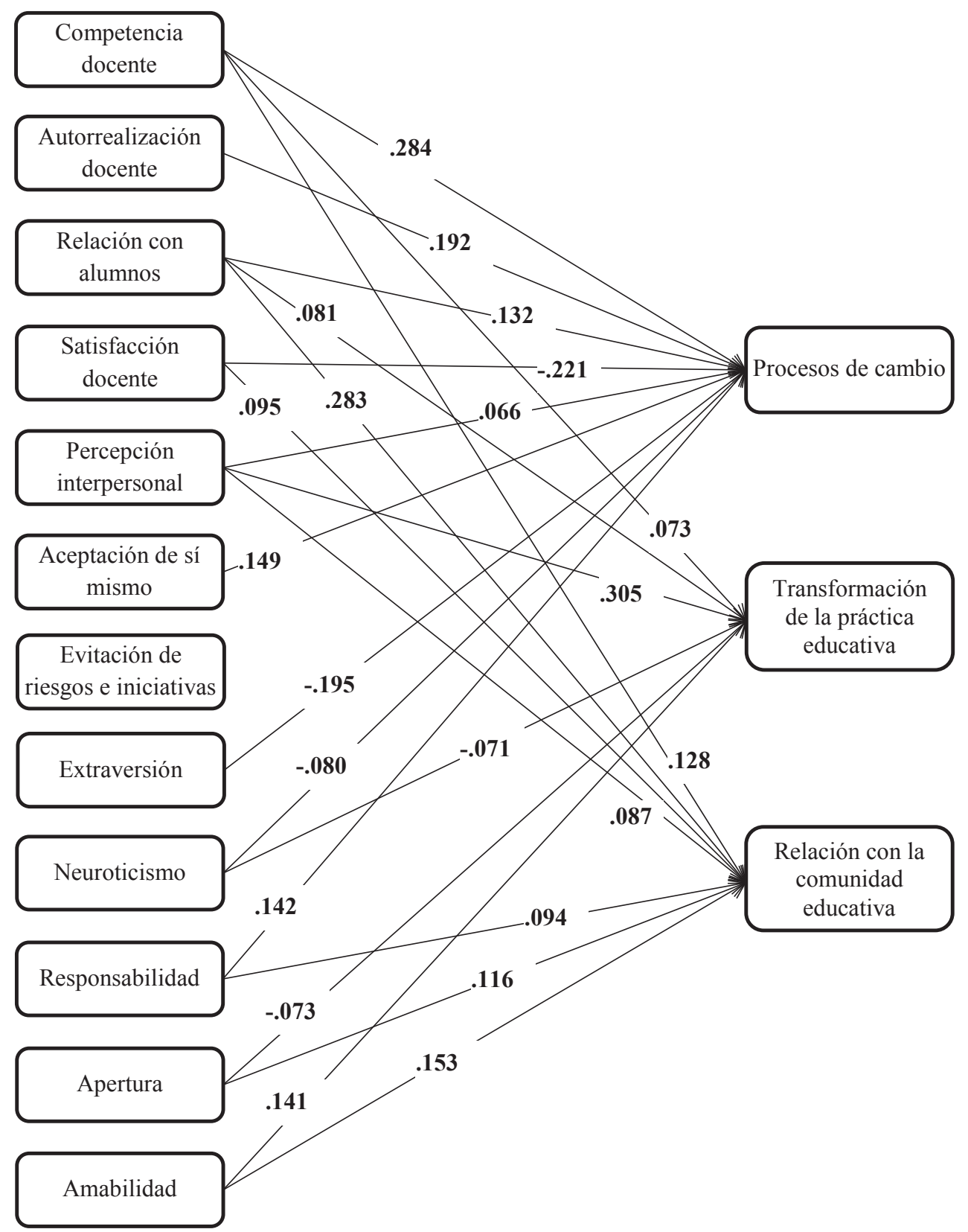

Figura 2. Modelo estructural final con los coeficientes estructurales estandarizados para predecir simultáneamente las tres medidas de Compromiso docente: Procesos de cambio, Transformación de la práctica educativa y Relación con la comunidad educativa. Nota: todas las estimaciones son estadísticamente significativas $(p<.05)$. 
El primer modelo estructural para predecir simultáneamente las tres medidas de Compromiso docente resultó excesivamente complejo. Si bien el ajuste global era razonablemente adecuado $\left(\mathrm{c}^{2}(3)=\right.$ 21.12, $\mathrm{p}<.001, \mathrm{RMSEA}=.085, \mathrm{SRMR}=$ .015 y $\mathrm{CFI}=.98)$, demasiadas relaciones resultaron estadísticamente no significativas. Por lo tanto, se procedió a fijar a cero los parámetros no significativos, y a volver a estimar y evaluar el modelo. El nuevo modelo, y la estimación estandarizada de sus parámetros, se presenta en la Figura 2. Alguna relación más resultó no significativa, lo que puede apreciarse en la correspondiente ausencia de flecha, pero ya no se realizaron cambios adicionales. De la misma manera, para simplificar el modelo, no se han explicitado las correlaciones entre predictores, que las hay y de cuantía importante, pero sí se presentan en la Tabla 1. Este modelo, más parsimonioso, también se ajusta de forma muy adecuada a los datos observados, como puede verse en los índices de ajuste: $\mathrm{c}^{2}(63)=68.17, \mathrm{p}<.001, \mathrm{RMSEA}=$ $.01, \mathrm{SRMR}=.023$ у $\mathrm{CFI}=.99$.

Los coeficientes estandarizados en la Figura 2 muestran la dirección y la fuerza de las relaciones encontradas entre los predictores y los criterios (las tres dimensiones del Compromiso docente). En cuanto a la capacidad predictiva conjunta que presentan todos los predictores sobre las tres dimensiones del Compromiso docente, esta se puede ver en los valores de la correlación múltiple al cuadrado. Estos valores de R-cuadrado fueron de .389 para el Compromiso con los procesos de cambio, de .142 para el
Compromiso con la transformación de la práctica educativa y de 179 para la relación con la comunidad.

\section{CONCLUSIONES Y DISCUSIÓN}

Se puso a prueba un modelo estructural con variables observables o path analysis para evaluar la capacidad predictiva sobre el compromiso docente. Se partió de la hipótesis de que determinadas características personales de los docentes influían en el ámbito personal y profesional de los mismos, y se diseñó un modelo SEM para poner a prueba la capacidad predictiva de dichas características.

Se estudió la capacidad predictiva de diferentes medidas de autoconcepto docente (competencia docente, autorrealización docente, relación con alumnos, satisfacción docente, percepción interpersonal, aceptación de sí mismo y evitación de riesgos e iniciativas) sobre el compromiso docente, definido por la relación con la comunidad educativa, el compromiso con los procesos de cambio y el compromiso con la transformación de la práctica educativa. También se examinó el poder predictivo de la personalidad, que comprendía neuroticismo, introversión, apertura, amabilidad y responsabilidad, sobre el compromiso docente.

Los procesos de cambio docente se vieron predichos en un $38,9 \%$ por todas las variables del autoconcepto, a excepción de la evitación de riesgos e iniciativas, la responsabilidad del docente, la estabilidad emocional y la extraversión. El compromiso con la transformación de la práctica educativa se predijo en un $14,2 \%$ por el sentido de competencia 
del docente, la amabilidad, una percepción interpersonal positiva, las relaciones profesor-alumno positivas, la satisfacción del docente, la estabilidad emocional y la falta de apertura (mínimamente). Finalmente, el compromiso docente en la relación con la comunidad educativa es predicho en un $17,9 \%$ por el sentido de competencia, la buena relación profesoralumno, la satisfacción del docente, la percepción interpersonal, la responsabilidad y la amabilidad.

En suma, el modelo confirma que variables personales, como son el autoconcepto y la personalidad, tienen poder predictivo sobre el compromiso docente. Por un lado, hay evidencia en torno a la idea de que las características de personalidad se vinculen con los roles laborales (Rapoport, 1997), puesto que en los resultados se puede observar que el rol de docente comprometido se identifica con la presencia de estabilidad emocional, extraversión y responsabilidad, tal como argumentan otros autores (Del Buey et al., 2008; Judge et al., 2002). Parece que, tal y como argumenta Peiró (1987), la personalidad del docente es importante respecto a la profesión, puesto que las características del profesor repercuten en los alumnos, y en concreto en el cambio educativo.

También es importante el autoconcepto. Concretamente, parece que, según lo señalado por la literatura (Gómez, 2007), el sentido de competencia del docente tiene un efecto en su labor. También juega un papel importante la percepción interpersonal, que apoya la idea de que, mediante las relaciones con otros, se obtiene información sobre uno mismo (Cava y Musitu, 2000), y, en el caso del docente, puede serle de ayuda para replantear su profesión. En cuanto a la relación profesor-alumno, se ha visto que esta tiene un efecto positivo en el alumno que potencia un rendimiento más alto en el profesor (Burns, 1990). Es posible que este aumento del rendimiento sea consecuencia de un mayor compromiso en el ámbito académico (Kompaso y Sridevi, 2010). De la misma forma, la satisfacción laboral del docente genera mayor rendimiento en este (Chamundeswari, 2013), lo que también puede verse como consecuencia de un mayor nivel de compromiso. Finalmente, como Naranjo (2007) argumenta, la aceptación o valoración positiva de uno mismo parece conducir a resultados positivos en el ámbito académico; entre estos, según los resultados obtenidos, destaca un mayor nivel de compromiso.

En conclusión, tanto la personalidad como el autoconcepto parecen ejercer influencia en el compromiso del docente con el cambio educativo. Este hallazgo tiene implicaciones en cuanto a la implementación de reformas educativas, puesto que, si se conocen los factores que propician el compromiso del personal docente con la reforma educativa, se podrán tomar medidas para que esta se lleve a cabo de forma efectiva.

La presente investigación presenta los resultados en una muestra representativa, $\mathrm{y}$ con un margen de error pequeño, de docentes peruanos. Esta es, sin duda, una de sus principales fortalezas. Presenta, no 
obstante, limitaciones. Una primera es el carácter transversal de los datos de estudio, que no permite realizar inferencias fuertes sobre causalidad más allá de las motivadas por razones teóricas. Que sean los docentes los únicos que han ofrecido información puede llevar también a algún grado de varianza común. Esto nos lleva a las previsiones de futuras direcciones en la investigación, que irían en la línea de la recogida de datos longitudinales, junto con la triangulación de informadores para eliminar potenciales sesgos y enriquecer la información.

\section{Referencias}

Bentler, P. M. (2005). EQS Structural Equations Program Manual. California: Multivariate Software, Inc.

Burns, R. (1990). El autoconcepto. Teoría, medición, desarrollo y comportamiento. Bilbao: Ega.

Calvete, E., y Villa, A. (1997). Programa “Deusto 14-16”. II. Evaluación e intervención en el estrés docente. Bilbao: Mensajero.

Cava, M., y Musitu, G. (2000). La potenciación de la autoestima en la escuela. Barcelona: Paidós Ibérica.

Chamundeswari, S. (2013). Job Satisfaction and Performance of School Teachers [Satisfacción laboral y rendimiento de los profesores en la escuela]. International Journal of Academic Research in Business and Social Sciences, 3(5), 420-428.

Consejo Nacional de Educación (2007). Proyecto Educativo Nacional al 2021.
La educación que queremos para el Perú. Lima: Ministerio de Educación.

Costa, P., y McCrae, R. (2008). NEO PI-R Inventario de Personalidad NEO Revisado. NEO-FFI Inventario NEO Reducido de Cinco Factores. Madrid: TEA Ediciones.

Del Buey, M. F., Fernández, A., Martín, E., Dapelo, B., Marcone, R., y Granados, P. (2008). Cuestionario de personalidad eficaz para la formación profesional. Psicothema, 20(2), 224-228.

Finney, S. J., y Di Stefano, C. (2006). Non-Normal and Categorical Data in SEM. En G. R. Hancock y R. O. Mueller (Eds.), Structural Equation Modelling: A second course (pp. 269314). Greenwich, CO: Information Age Publishing.

Gómez, L. (2007). Desarrollo humano del profesorado. Módulo del Programa de Doctorado 700H Psicología de la Educación y Desarrollo Humano de la Universitat de València. Lima: ISPP Crea.

Hu, L., y Bentler, P. M. (1999). Cut-Off Criteria for Fit Indexes in Covariance Structure Analysis: Conventional Criteria Versus New Alternatives. Structural Equation Modeling, 6, 1-55.

Judge, T., Heller, D., y Mount, M. (2002). Five Factor Model of Personality and Job Satisfaction. A Meta-Analysis. Journal of Applied Psychology, 87(3), 530-541.

Kompaso, S., y Sridevi, M. (2010). EmployeeEngagement: TheKey to Improving Performance [Compromiso 
de los empleados: la clave para mejorar el rendimiento]. International Journal of Business and Management, 5(12), 89-96.

Llor, B., Abad, M.A., García, M., y Nieto, J. (1995). Ciencias psicosociales aplicadas a la salud. Madrid: McGraw-Hill Interamericana de España.

Martínez, A. (2008). Metodología de la investigación educativa. Módulo del Programa de Doctorado 700H Psicología de la Educación y Desarrollo Humano de la Universitat de València. Lima: ISPP Crea.

Monereo, C. (2010). ¡Saquen el libro de texto! Resistencia, obstáculos y alternativas en la formación de los docentes para el cambio educativo. Revista de Educación, 352(1), 583-597.

Naranjo, M. L. (2007). Autoestima: un factor relevante en la vida de la persona y tema esencial del proceso educativo. Actualidades Investigativas en Educación, 7(3), 1-27.

Peiró, J. M. (1987). Estudio psicosocial de la demanda y uso de las instalaciones deportivas en la Comunidad Valenciana (I). Informe realizado para la Consellería de Cultura, Educación y Ciencia. Valencia.

Rapoport, A. (1997). Science, Explanatory Theory and Environment-Behavior Studies. En S. Wapner, J.
Demick, T. Yamamoto y H. Minami (Eds.), Theoretical Perspectives in Environment-Behavior Research: Underlying Asumptions, ResearchProblems, and Methodologies (pp. 107- 140). New York: Plenum.

Sánchez, C., y Cerviño, C. (2006). Factores que condicionan los niveles de satisfacción del docente dominicano y su influencia en el compromiso con los procesos de cambio y transformación de la práctica educativa. Trabajo de Investigación. Valencia: Publicaciones de la Universidad Autónoma de Santo Domingo y Universitat de València.

Tanaka, J. S. (1993). Multifaceted Conceptions of Fit in Structural Equation Models. En K. A. Bollen y J. S. Long (Eds.), Testing structural equation models (pp. 10-39). Newbury Park, CA: Sage.

Tedesco, J. C., y López, N. (2013). Diez años después. Comentarios tras una relectura del artículo "Algunos dilemas de la educación secundaria en América Latina". Revista Iberoamericana sobre Calidad, Eficacia y Cambio en Educación, 11(2), 10-32.

Tovar, T. (2009). El Proyecto Educativo Nacional: punto de partida, punto de quiebre y punto de llegada. En R. Cuenca (Coord.), La educación en los tiempos del APRA: Balance 2006-2009 (pp. 143-166). Lima: Foro Educativo. 